\title{
$B M J$ Quality Reducing the number of unnecessary liver function tests requested on the Paediatric Intensive Care Unit
}

\author{
Lynn Sinitsky, Joe Brierley
}

To cite: Sinitsky L, Brierley J. Reducing the number of unnecessary liver function tests requested on the Paediatric Intensive Care Unit. BMJ Quality Improvement Reports 2017;6:u214071. w5561. doi:10.1136/ bmjquality.u214071.w5561

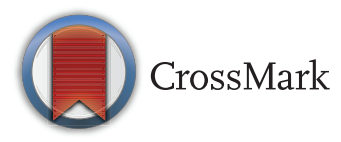

Great Ormond Street Hospital for Children, UK

\section{ABSTRACT}

Between January and October 2014, Great Ormond Street Hospital Paediatric Intensive Care Unit (PICU) was spending an average £23,392 on blood tests per month. Blood tests should be requested based on previous results and the patient's clinical condition, medication and nutritional status. However, more blood tests were being ordered than clinically indicated: an audit in October 2014 showed liver function tests (LFTs) were requested daily on most patients, even with previous normal results.

A driver diagram identified three primary drivers for blood test requesting: decision-making, situational awareness and computer-based ordering. Decisionmaking for routine blood tests was the responsibility of the bedside nurses on each night shift. The communication between the nurses and doctors was an identified secondary driver. The project's primary aim was to reduce unnecessary LFTs requests on PICU over 6 months by implementing a blood test request form, a table of common investigations to facilitate and document discussion between the nursing and medical teams. The secondary aims were to reduce other unnecessary blood test requests, including full blood counts (FBC), coagulation screens and CRP.

This project was conducted in three phases: construction, testing and implementation of the blood test form. PDSA cycles were used within each phase. Two PICU nurse champions were engaged to provide bedside support, education and feedback.

In the 8-month period following implementation, there was a significant sustained reduction in LFTs requests. A similar pattern of sustained reduction also occurred for FBC, coagulation screens and CRP requests. This sustained reduction in blood tests requested equated to a saving in excess of $£ 36,000$.

This project was successful: the reduction in the number of inappropriate blood tests had clear financial benefit for PICU and reduced blood loss for patients. Early engagement and support from key stakeholders avoided conflict, guaranteed data sharing and aided engagement of bedside nurses.

\section{PROBLEM}

The Paediatric Intensive Care Unit (PICU) in Great Ormond Street Hospital for Children (GOSH) admits approximately
1,200 patients each year, predominately from the North Thames area but also from all over the UK and abroad, reflecting the wide range of specialist services that the unit provides. Admission to an intensive care bed is costly and expenditure on biochemical, haematological, and bacteriologic tests, important for diagnosis and monitoring in the critically ill, are included in the variable costs accrued according to individual need. ${ }^{1}$

The Great Ormond Street Hospital for Children NHS Foundation Trust is currently experiencing significant financial pressures, similar to many UK Trusts, due to changes in commissioning strategies, limits in growth of specialised commissioning budgets, reduced tariffs for specialised services and increased costs to deliver new regulatory requirements. ${ }^{2}$ Therefore one priority in the Annual Plan for $2015 / 16$ is the emphasis on increasing the Productivity and Efficiency (Cost Improvement) Programme across the Trust. "No waste", eliminating waste by ensuring effective and efficient care, became one of the four strategic initiatives for the service redesign project facilitated by the Trust Quality Improvement team. ${ }^{3}$

\section{BACKGROUND}

At least $20 \%$ of healthcare spending globally is wasted. ${ }^{12}$ In the NHS, overuse of diagnostic or monitoring tests is a key example of wasted resources ${ }^{3}$. Between January and October 2014 PICU was spending an average $£ 23,392$ on blood tests per month, with liver function tests (LFTs) costing up to $£ 3000$ per month.

In October 2014 a survey of the PICU consultants identified a consensus advocating liver function tests should be sent on an individual patient specific basis both on admission and throughout patient stay. If a patient had normal liver function and there was no potential for change, frequent repeating of this blood test panel was unnecessary as it 
would be expected to remain within normal parameters. If a patient had abnormal liver function, or there was potential for normal liver function to become deranged secondary to the underlying clinical condition or administration of medication or parental nutrition, repeating this blood test was considered clinically indicated but the frequency should be reviewed regularly.

In the same month, an audit of the number of liver function tests requested for 12 PICU inpatients during a 7 day period was conducted. The appropriateness of each blood test was evaluated according to the patient's clinical condition, medication, nutritional status and previous pathology results, with particular regard to the results from the consultant survey. The audit showed LFTs continued to be requested daily in all patients after an initial normal result. In one patient, 22 LFTs had been taken in the 7 day audit period, at a cost of $£ 137.50$, with all the results being within normal parameters. The baseline data showed that there were many, clinically unnecessary LFTs being requested on PICU. Reducing the number of unnecessary blood tests would have clear financial benefit for the PICU and would also reduce blood loss for patients.

There is evidence that patient care, clinical outcomes and reduction in the use of resources can be achieved by doctors at any level of training or speciality tackling this and other key areas, such as overuse of medication and unplanned admissions, with quality improvement initiatives ${ }^{3}$. Successful strategies to reduce the number of unnecessary blood tests requested include proformas that limit or guide blood testing requests, ${ }^{45}$ provision of price information at the point of requesting, ${ }^{6} 7$ and using senior clinicians to vet junior doctor pathology requests. $^{8}$

A driver diagram identified three primary drivers for blood test requesting: decision-making, situational awareness and computer-based ordering. In PICU decisionmaking for routine blood tests was the responsibility of the bedside nurses on each night shift. The communication between the nurses and doctors was an identified secondary driver.

The project's SMART aim was reduce the number of LFTs requested over 6 months to below the background mean by implementing a blood test request form. The blood test form was a table of common investigations that would facilitate and document discussion between the nursing and medical teams about individual patient pathology requests thereby aiding nurse decision-making on blood tests requests. The secondary aim was to reduce PICU requests of other unnecessary blood test, including full blood counts, coagulation screens and CRP.

\section{BASELINE MEASUREMENT}

Monthly data on the total number and cost of each blood test type requested by each ward was available from the haematology and biochemistry departments as this data regularly fed into Trust governance and transformation workstreams. Therefore the study used outcome measures available from this data. Data from January to October 2014 was used to generate baseline measurement and construct SPC charts for each blood test type. The primary outcome measure was the total number of LFTs each month per PICU bed days. The secondary outcome measures were the total number of full blood counts, coagulation screens and CRP requests each month per PICU bed days. Monthly data of laboratory costs for each blood type as well as total spending was also collected.

\section{DESIGN}

Poor communication between the nursing and medical teams was identified as a potential problem contributing to the large number of unnecessary blood tests being requested. The night PICU bedside nurses were responsible for routine blood test requests. There were no formal discussions regarding routine investigations with the medical team during the evening ward round. Therefore, the nurse's experience and knowledge may limit his/her ability to make appropriate judgements regarding applicability and frequency of specific blood tests, and so the "default" state would often be to request every test.

The project aimed to reduce the number of LFTs requested by implementing a form to improve communication between the nursing and medical teams. The blood test request form listed common laboratory tests. During the bedside evening ward round, the form guided discussion between nursing and medical staff on which investigations were to be requested that shift for each patient. In addition, the form provided formal documentation of agreed required tests.

A QI team consisting of a PICU Junior Doctor (project lead), Service Manager for Critical Care, Senior Analyst from the Quality Improvement team, two PICU nurse champions and important stakeholders including the PICU lead nurse, PICU physician champions and laboratory managers was formed. Engagement and support from the PICU lead nurse and consultants enabled a change in the night ward round structure and the introduction of a new form. The PICU nursing and medical staff were encouraged to participate in the project and change their working practice. Initial requests for sharing of laboratory data (number of requests and costs) were met with reluctance. Involvement of the Service Manager for Critical Care aided communication with the laboratory manager and facilitated an agreement for regular sharing of monthly laboratory data as the project clearly aligned with the "no waste" Trust aim.

\section{STRATEGY}

The Project was conducted in three phases: construction, testing and implementation of the blood test form. PDSA cycles were used within each phase. Throughout 
phases 1 and 2, awareness of the project was raised with posters, emails and departmental presentations. Two PICU nurse champions were engaged to support bedside nursing education and collect and share feedback.

Phase 1: Construction of blood test form

In the first phase of this project the blood test request form was designed. Each version of the form was sent firstly to the PICU lead nurse and then to the PICU consultants. Changes were made based on written feedback until the final version was signed off by both senior stakeholders.

Phase 2: Testing of blood test form

In the second phase, the first PDSA cycle tested the form on one night shift. The PICU nurse in charge and night doctor for that shift gave positive feedback on the format of the form and its ease of use. Testing continued on successive night shifts for a two week period with feedback sought each morning. Each night shift therefore became the next PDSA cycle. Running out of forms or forgetting to collect a form at the start of the shift were the main problems encountered. After the first week, the PICU administrative team began stapling the form to the infusion worksheet used on every shift. Subsequently there were no problems with accessibility.

Phase 3: Implementation of blood test form

In the first PDSA cycle of the third phase, the blood test form was used on every night shift. Outcome measures after the first month showed a reduction in the number of blood test requests. However, a survey of the nurses at the end of the first month found that the form was not used in $50 \%$ of shifts, predominantly because the form did not include options such as "no bloods needed".
This feedback led to a change in form design. In the second PDSA cycle the new form was stapled to the infusion worksheet. In addition, stickers were placed at every bedside computer to act as a reminder.

After each month, the number of blood requests (LFTs, FBC, coagulation screens and CRP) and pathology spending were disseminated to staff by email, posters and at departmental presentations. Staff were regularly thanked and encouraged to sustain their achievements.

\section{RESULTS}

In the 8 months after this project started, there was sustained reduction in LFTs requested on PICU as shown in Figure 1(a). A similar pattern of reduction in blood test requests occurred for full blood counts, coagulation screens and CRP as shown in Figures 1(b), 1(c) and 1 (d). For all four of the biochemical and haematological blood tests investigated, there was a reduction to below the lower confidence level after the second month of implementation, followed by a general plateau with variability at a new lower mean.

This project was successful. In the 9 months from the outset of the project, November 2014 to July 2015, PICU spent approximately £4000-5000 less each month on blood tests. This equates to a saving in excess of $£ 36,000$ over that period.

\section{LESSONS AND LIMITATIONS}

There were two important lessons learnt during this project. The first was the importance of early identification and engagement of key stakeholders. Without them
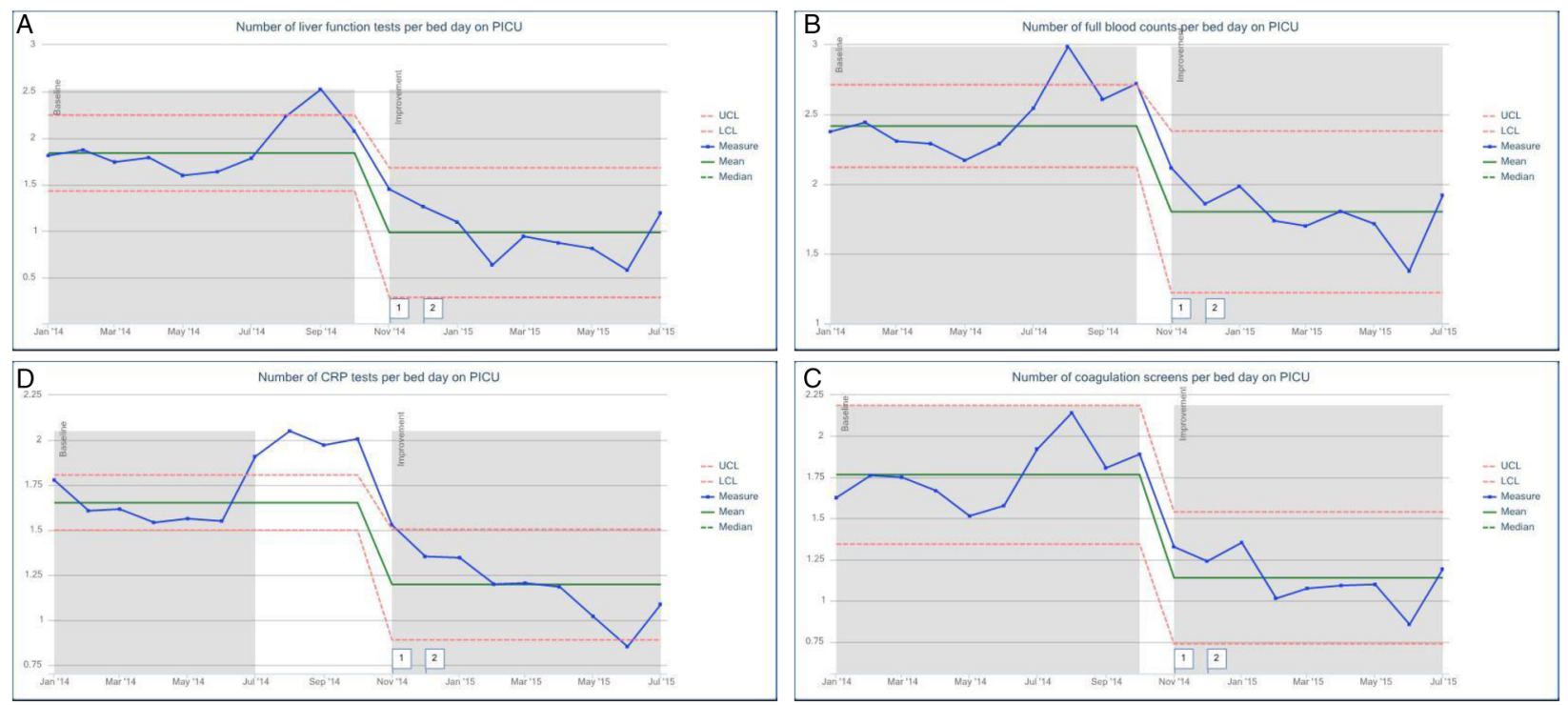

(a) Number of liver function tests per PICU bed day (c) Number of CRP tests per PICU bed day

(b) Number of full blood counts per PICU bed day

[1] Start of phase two - testing

(d) Number of coagulation screens per PICU bed day

[2] Start of phase three - implementation

Figure 1 
the project could not have been realised. Engagement and collaboration with the Critical Care manager avoided conflict with the laboratory manager and led to an agreement for regular data sharing. Support of senior clinical stakeholders facilitated changes and teamwork at the ward level. The second was having PICU nurse champions "on the ground" from the outset of the project. They helped with bedside nursing support and education, collected feedback and communicated changes and results. This personal approach ensured all the nursing staff felt listened to and their contributions and actions valued. The PICU nurses took ownership of their change in practice; they were proud of their success and so motivated to engage more. Over six months, the project team reduced the level of bedside support and education, but the reduction in the number of blood test requests continued. The intervention had led to a sustainable change in practice, a new "culture" of improved communication and consideration regarding blood test requests. Furthermore, to sustain this change going forward, the PICU nurse practice educators planned to include more information on investigations, for example rationale and expected frequency for requests, within monthly teaching topics. Nurses' feedback had requested more focused learning on pathology investigation as greater clinical understanding would help their decision-making abilities.

Although this project's intervention had minimal financial cost, significant time was invested by the project lead and nurse champions in the initial months to facilitate education sessions and collect and respond to feedback. The sustained reduction in the number of blood test requests beyond the project's six month aim resulted in large financial savings for the unit, making this an overall very cost-effective intervention.

Blood tests requests are very common and so there is huge potential for waste reduction in all healthcare areas. The project aim of reducing unnecessary blood tests is applicable to all, but the intervention used in this project may not be generalisable. Different wards/units may have different processes for how and who requests pathology tests. This project implemented an intervention to improve nurse-doctor communication and aid nurse decision-making as they were the primary requestors. If nurses have no role in blood tests requesting, a different intervention will be needed as guided by the drivers of the blood test process in any specific department.

Our study did not include data on the clinical conditions of the PICU patients admitted during the study period. Specific clinical conditions may have confounded the data. For example, bronchiolitis is a primarily respiratory seasonal condition which would not require routine blood test monitoring. Over the winter months of the study, when bronchiolitis would be expected to dominate PICU inpatient admissions, a reduction in blood test requests would be expected. However, the reported results span 9 months from winter into summer and show a significant sustained reduction to below the lower confidence level throughout this period which cannot be explained by chance or potential confounding conditions.

\section{CONCLUSION}

This project was successful: after the implementation of the blood test request form, there was a sustained reduction in the number of blood test requests with clear financial benefit for the PICU. From the baseline data, it was clear there were many unnecessary LFTs being ordered on the PICU and so the reduction in liver function test requests achieved in the first few months of the project was predictable. However, the reduction in the other biochemical and haematological tests was surprising as it showed a lot more waste in the system than expected.

Although the intervention was successful in achieving the project's aim, the blood test request form alone did not change practice. In the first month of implementation the form was used in less than $50 \%$ of shifts but there was still a significant reduction in all blood test requests. The blood test request form was a tool: it encouraged staff to question their clinical practice, opened discussions regarding the clinical indication for investigations, encouraged multi-disciplinary communication and raised awareness about the cost of critical care. This echoed the findings shown by Mughal et al. ${ }^{11}$ that unnecessary blood tests requests can be reduced by introducing discussion between junior and senior clinicians to direct decision-making and ensure tests are requested based on clinical need. Overall the intervention resulted in a sustained change in practice from a generalised approach to blood test requests to more patient-centred individualised care. This individualised approach is in contrast to other studies which have shown reduction of unnecessary tests by using pre-set pathology request proformas. ${ }^{9} 10$

It was important to be mindful during this project that important tests could be missed by encouraging less blood tests to be requested. Balancing measures were introduced to minimise potential harm. There were teaching sessions and board displays on the blood tests indicated in different clinical conditions commonly managed on the PICU. A reporting process was also set up whereby the PICU consultants could feedback any concerns identified after their daily review of blood results. During the 9 month study period no major concerns were raised.

Requesting too many, not clinically indicated blood tests, is not a phenomenon specific to PICU. In children with indwelling central venous or arterial lines, blood testing is painless and easy and so frequent blood test requests are common. Following on from the success of this project on the PICU and its alignment with Trust aims, the Critical Care Senior Manager began engaging key senior clinical stakeholders across the Critical Care 
directorate, specifically from the Neonatal and Cardiac Intensive Care Units, to initiate similar interventions and reduce unnecessary blood tests in their department.

Acknowledgements Sarah Barton, Ivan Brooke, Mike Coochneea, Duncan Shephard, Ghil Stephonson and the Great Ormond Street Hospital EQulP Quality Improvement course facilitators.

Declaration of interests Nothing to declare.

Ethical approval According to the policy activities that constitute research at the Great Ormond Street Hospital for Children NHS Foundation Trust, this work met criteria for operational improvement activities excempt from ethics review.

Open Access This is an open-access article distributed under the terms of the Creative Commons Attribution Non-commercial License, which permits use, distribution, and reproduction in any medium, provided the original work is properly cited, the use is non commercial and is otherwise in compliance with the license. See:

- $h t t p: / / c r e a t i v e c o m m o n s . o r g / l i c e n s e s / b y-n c / 2.0 /$

- http://creativecommons.org/licenses/by-nc/2.0/legalcode

\section{REFERENCES}

1. García S, Ruza F, Alvarado F, et al. Analysis of costs in a pediatric ICU. Intensive Care Med. 1997;23:218-25.
2. Annual report and accounts $2014 / 14$. Great Ormond Street Hospital for Children NHS Foundation Trust. http://issuu.com/goshcc/docs/ 15_05_annual_reports_hospital_repor/174? e=1 [last accessed 23/ 06/16]

3. Quality improvement. Great Ormond Street Hospital for Children NHS Foundation Trust. http://www.gosh.nhs.uk/about-us/ our-priorities/quality-improvement [last accessed 23/06/16]

4. Organisation for Economic Cooperation and Development. Tackling wasteful spending on health. OECD 2017.

5. World Health Organization. World health report: health systems financing: the path to universal coverage. WHO 2010.

6. Academy of Royal Medical Colleges. Protecting Resources Promoting Value. A Doctors Guide to Cutting Waste in Clinical Care. 6th November 2014.

7. Akhtar W, Chung Y. Saving the NHS one blood test at a time. BMJ Qual Improv Rep. 2014;2.

8. Chu KH, Wagholikar AS, Greenslade JH, O'Dwyer JA, Brown AF Sustained reductions in emergency department laboratory test orders: impact of a simple intervention. Postgrad Med J. 2013;89:566-71.

9. Hampers LC, Cha S, Gutglass DJ, Krug SE, Binns HJ. The effect of price information on test-ordering behavior and patient outcomes in a pediatric emergency department. Pediatrics. 1999;103:877-82.

10. Feldman LS, Shihab HM, Thiemann D, et al. Impact of providing fee data on laboratory test ordering: a controlled clinical trial. JAMA Intern Med. 2013;173:903-8.

11. Mughal Z, Narayanan A, Gupta V, Reay-Jones N. Clinical need-directed blood tests: a step in saving the NHS? Ann Clin Biochem.2016;53:568-74. 